\title{
CITIZEN RELATIONSHIP MANAGMENT: Proposta para Prefeituras realizarem a gestão do relacionamento com os cidadãos, apoiada por Sistemas de Informação integrados.
}

\author{
Leonardo Oliveira Leite ${ }^{1}$, Denis Alcides Rezende ${ }^{2}$ \\ ${ }^{1}$ Pontifícia Universidade Católica do Paraná - Mestrado em Gestão Urbana \\ ${ }^{2}$ Pontifícia Universidade Católica do Paraná - Mestrado em Gestão Urbana \\ leonardo.leite@serpro.gov.br, denis.rezende@pucpr.br
}

\begin{abstract}
Resumo. As Prefeituras mostram grandes dificuldades no atendimento efetivo de seus cidadãos. $O$ objetivo deste trabalho é adaptar o conceito de Costumer Relationship Management (CRM), já difundido na iniciativa privada, para Prefeituras, promovendo a melhoria dos serviços públicos e a aproximação do gestor público ao cidadão. O método de pesquisa bibliográfica exploratória utilizado neste artigo, propõe a implantação de um CzRM Municipal, baseado na integração dos Sistemas de Informação. A conclusão reitera que o CzRM permitirá às Prefeituras conhecer seus cidadãos, personalizando o atendimento, apoiando o processo decisório e otimizando a máquina pública para atender de forma mais efetiva seus cidadãos.
\end{abstract}

\section{Introdução}

A gestão municipal é uma tarefa extremamente desafiadora. Abrange a necessidade de conciliar os mais diversos interesses e de atingir resultados com grande complexidade de mensuração, dispondo para isso de recursos e estrutura limitados, muitas vezes obsoletos; e também poucas informações adequadas e oportunas para as decisões.

A iniciativa privada enfrentou problemas similares e formulou alternativas para obter destaque entre seus concorrentes, por meio do conhecimento e da aproximação dos clientes, o que foi possível a partir da utilização das Tecnologias de Informação e Comunicação (TICs), para gerir e realizar seus relacionamentos com os clientes. Neste cenário, os Sistemas de Informação (SI) tem papel fundamental, pois podem viabilizar a estruturação, a armazenagem, a recuperação e a operacionalização de grandes volumes de dados dos clientes na iniciativa provadas e dos cidadãos na iniciativa pública.

O objetivo deste trabalho é adaptar os conceitos de Customer Relationship Management (CRM) difundidos na iniciativa privada, para o ambiente público de Prefeituras, promovendo a melhoria dos serviços públicos prestados e a aproximação do gestor público ao cidadão. É com este intuito que é proposta a estruturação de um Citizen Relationship Management (CzRM) para uma administração pública municipal, baseada na integração dos Sistemas de Informação (SI) existentes nas Prefeituras. 


\section{Fundamentação Teórica}

\subsection{Customer Relationship Management (CRM)}

Nos anos 90, com um ambiente internacional extremamente competitivo, passou a ser essencial para as empresas o conhecimento e o entendimento de seus consumidores. O Customer Relationship Management (CRM) é um conceito bastante difundido entre as organizações privadas, nestes casos focando principalmente a fidelização dos clientes e a maximização do lucro; conforme define Swift: “é um processo interativo que transforma informações sobre clientes em relacionamentos positivos com os mesmos”. As empresas buscam identificar o cliente, conhecê-lo, diferenciar (avaliando as suas necessidades e exigências), interagir com ele (reavaliando suas necessidades) e personalizar este relacionamento (SWIFT, 2001).

O CRM deve ser integrado a tudo que a empresa faz, a todos com os quais ela trabalha e transaciona. O foco da empresa é mudado de seu produto ou serviço para o cliente, mudando também a cultura desta empresa. Além disso, as comunicações devem ser da mão dupla, integradas, registradas e gerenciadas, pois sem dados históricos, transações detalhadas, comunicações focalizadas e categorizadas, um relacionamento não pode ser efetivamente mantido (ZENONE, 2001).

O papel da Tecnologia de Informação e Comunicação (TIC) é essencial neste cenário, pois ela é que possibilitará o tratamento das informações e processos que facilitem o trabalho da empresa na coleta e no gerenciamento das informações sobre os clientes e o negócio, permitindo a transformação de informações em conhecimento (SWIFT, 2001).

\subsection{New Public Management - NPM}

A nova gestão pública ou a "new public management” (NPM) pressupõe aplicar nas organizações públicas os modelos de gestão originalmente oriundos da iniciativa privada e dos conceitos de administração estratégica focada nos negócios empresariais e nos conceitos de empreendedorismo. A administração pública passa por um momento de repensar seu papel frente à sociedade, mudando seu objetivo, que antes era visto como o de controlar os rumos da sociedade, para uma visão onde ela procura auxiliar os cidadãos no atendimento de seus interesses. Com esta mudança, a administração pública precisou repensar o modus-operandi dos processos produtivos ora existentes no Governo e buscar formas de melhorar seus processos internos e externos, colocando a satisfação do cidadão como um dos principais objetivos a serem alcançados. Na administração pública municipal esta transformação é sentida com mais intensidade, pois é o Poder Público mais "próximo" do cidadão e que possui a incumbência de gerir a infra-estrutura das cidades, estando intimamente relacionado com a qualidade de vida de seus cidadãos (REZENDE; CASTOR, 2006).

A NPM apresenta como características: contextualizar o cidadão como um cliente em foco; dar o sentido claro da missão da organização pública; delegar autoridades; substituir normas por incentivos; elaborar orçamentos baseados em resultados; expor operações do governo à concorrência; procurar soluções de mercado e não apenas administrativas; e medir o sucesso do governo pelo cidadão. Também tem como princípios: reestruturação; reengenharia; reinvenção; realinhamento; e reconceituação (JONES; THOMPSON, 2000). 
Como uma alternativa na busca pela transposição destes desafios surgiu à aplicação de modelos de gestão da iniciativa privada na administração pública e a utilização das novas tecnologias na transformação e na modernização da gestão pública, especialmente a municipal. A esta nova "doutrina” foi atribuído o nome de New Public Managment (NPM), ou Nova Administração Pública. A NPM traz ainda os conceitos de que a Administração Pública deve contextualizar o cidadão como cliente em foco e de que o gestor público deve se posicionar como um empresário e agir de forma empreendedora colocando em prática o que é planejado, gerando resultados positivos e para isto utilizando ferramentas e tecnologias disponíveis para auxiliá-lo (OSBORNE; GAEBLER, 1992; BARZELAY, 2001; REZENDE; CASTOR, 2006).

\section{Metodologia da pesquisa}

Foi realizada uma pesquisa bibliográfica exploratória de documentos eletrônicos, livros e periódicos (GIL, 2002). Buscou-se a fundamentação conceitual sobre CRM e NPM, a qual resultou na construção de hipóteses e na proposição de uma estruturação de um CzRM para a administração pública municipal. A metodologia para modelar a estrutura de CzRM para uma administração pública contemplou as seguintes fases: constituição das bases teórico-conceituais; estudo das estruturas de administrações públicas municipais; estudo da Central 156 e seu funcionamento na Cidade de Curitiba; estudo da adaptação do CRM ao ambiente público; e, documentação dos resultados.

A metodologia da pesquisa possui grande ênfase no método indutivo favorecido pelas experiências vivenciadas pelos autores e apresenta formas de satisfazer a necessidade de informações dos gestores públicos municipais, enquadrando-se, portanto, na qualificação de pesquisa qualitativa (LAKATOS; MARCONI, 1991).

A pesquisa tem objetivo prático para Prefeituras, portanto, pode ser considerada uma pesquisa aplicada (SILVA; MENEZES, 2001). Dessa forma, abrange uma visão de multimétodos para atender os objetivos propostos, mesclando abordagens e integrando instrumentos de pesquisa indissociáveis (NACHMIAS; NACHMIAS, 1987; VERGARA, 2005).

\section{Citizen Relationship Management (CzRM) Municipal}

Esta seção enfatiza a proposta para as Prefeituras realizarem a gestão do relacionamento com os cidadãos apoiada por sistemas de informação integrados. Para tanto, adapta-se os conceitos e a estruturação do CRM da iniciativa privada para o ambiente público municipal, começando pelo próprio acrônimo que a partir daqui passa a ser utilizado CzRM, significando Citizen Relationship Management, sendo esta adaptação também encontrada por CiRM ou Gestão de Relacionamento com os

Cidadãos. A implantação de um CRM Municipal significa para a administração pública municipal colocar os cidadãos e o relacionamento com os mesmos como o foco principal de sua atuação. (SCHELLONG, 2006).

\subsection{Processo interativo do CzRM Municipal}

Com base em Swift (2001) o CzRM deve ser visto pela Prefeitura como um processo interativo, um ciclo que envolve toda a sua estrutura com 4 fases (Figura 1): 


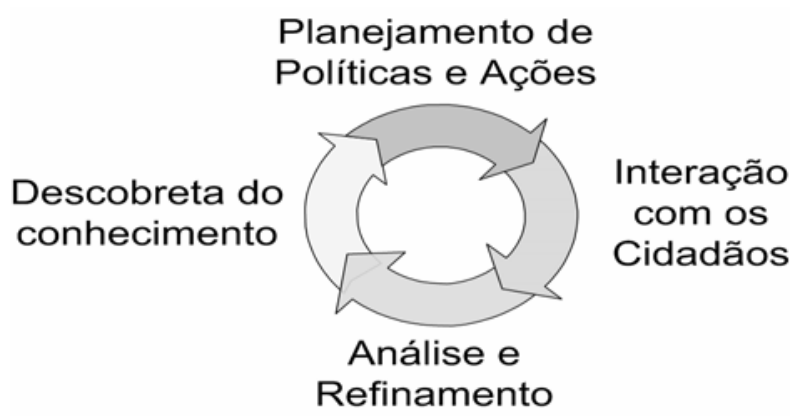

Figura 1. Fases do CzRM (SWIFT, 2001)

- Descoberta do conhecimento: é a análise das informações do cidadão para identificar suas expectativas em relação a sua cidade, suas demandas, necessidades, capacitação, situação econômico-social. Todos os dados, relações e transações deste cidadão precisam ser contextualizados e transformados em informações e conhecimentos.

- Planejamento de políticas e ações municipais: aqui são definidos os planos, programas e infra-estrutura necessários para atender, elaborar e/ou conciliar o planejamento do gestor municipal aos anseios da população.

- Interação com os cidadãos: nesta fase se gerem as comunicações com os cidadãos. Seja para levantar novas demandas e necessidades ou avaliar o sucesso e o impacto de determinado projeto, programa ou obra executados, ou para auxiliar no planejamento para futuros projetos, e até mesmo para levantar indicadores da satisfação da população. Aqui também estão inseridas as mobilizações da população, como por exemplo, um convite para a inauguração de uma obra ou para participação nos debates para a definição da Lei de Diretrizes Orçamentárias (LDO).

- Análise e refinamento: é a fase de aprendizagem contínua a partir das interações com os cidadãos. Aqui processos e serviços que não estejam atendendo aos anseios da população são revistos e reestruturados, impactando em toda a máquina da administração pública municipal, que estará buscando otimizar seus processos do ponto de vista do cidadão, pois é ele o ator central nesta forma de gestão.

Dados de relacionamentos entre a Prefeitura e os cidadãos já existem e estão dispersos entre os diversos Sistemas de Informação que a Prefeitura possui, em maior ou menor grau, dependendo do nível de informatização em que a Prefeitura se encontre. Como por exemplo, o Imposto Predial e Territorial Urbano (IPTU), o Imposto Sobre Serviços (ISS), atendimentos em postos de saúde, infrações de trânsito, demandas e solicitações registradas no Serviço 156, cadastros de escolas municipais, entre diversas outras bases de dados existentes no município. O grande desafio dos Sistemas de Informação é relacionar e comunicar estas diversas bases de dados para responderem de forma integrada. Além disso, os Sistemas de Informação tem a função de serem os responsáveis pela consolidação das informações disponíveis a serem apresentadas aos gestores públicos, apoiando seu processo de tomada de decisão.

Os Sistemas de Informação, e mais especificamente as Tecnologias de Informação e Comunicação, também tem papel importante no estabelecimento de 
interação e comunicação com os cidadãos, representada pela Internet, calls centers, quiosques de auto-atendimento, entre outros.

\subsection{Proposta de CzRM Municipal}

A Figura 2 representa como pode ser estruturado um CzRM Municipal. Na proposta existem três centrais que interagem diretamente com o cidadão.

- Central 156: é um serviço de utilidade pública, já regulamentado pela Agência Nacional de Telecomunicações (ANATEL) e destinado às Administrações Municipais. Já opera em diversos municípios brasileiros e tem por objetivo o atendimento da demanda de informações e solicitações dos munícipes. É essencialmente um call center receptivo.

- Central de relacionamento: permite gerir e comunicar as ações da Administração Pública nas mais diversas áreas, bem como, os Projetos e Serviços Prestados. É um call center ativo que realiza a divulgação e convites para eventos como inaugurações, campanhas e novos projetos da Prefeitura, além de contatos direcionados aos participantes dos Programas e Cursos da Prefeitura com intuito de manter um cadastro constante para convite e divulgação de novos eventos.

- Central de pesquisas: é um call center ativo que realiza pesquisas telefônicas junto aos usuários dos serviços da Administração Pública, realizando a avaliação dos projetos e programas disponibilizados para a população. Também pode realizar pesquisas de opinião pública para apoio a tomada de decisão, sobre temas polêmicos e a satisfação dos cidadãos em relação aos serviços públicos e a administração.

Estas centrais estão representadas separadamente, mas podem ser agrupadas ou integradas, desde que cumpram todas as funções descritas anteriormente. Elas alimentam suas bases de dados, que estão integradas entre si e também integradas com as demais bases de dados existentes no município, gerando uma grande base de informações que podem ser transformadas em conhecimento sobre os cidadãos do município. Além disso, a integração destas diferentes bases permite um atendimento personalizado aos cidadãos, sabendo rapidamente suas demandas e o histórico de relacionamento deste cidadão com a administração pública municipal e possibilitando fomentar uma maior participação deste cidadão na melhoria dos serviços públicos municipais. 


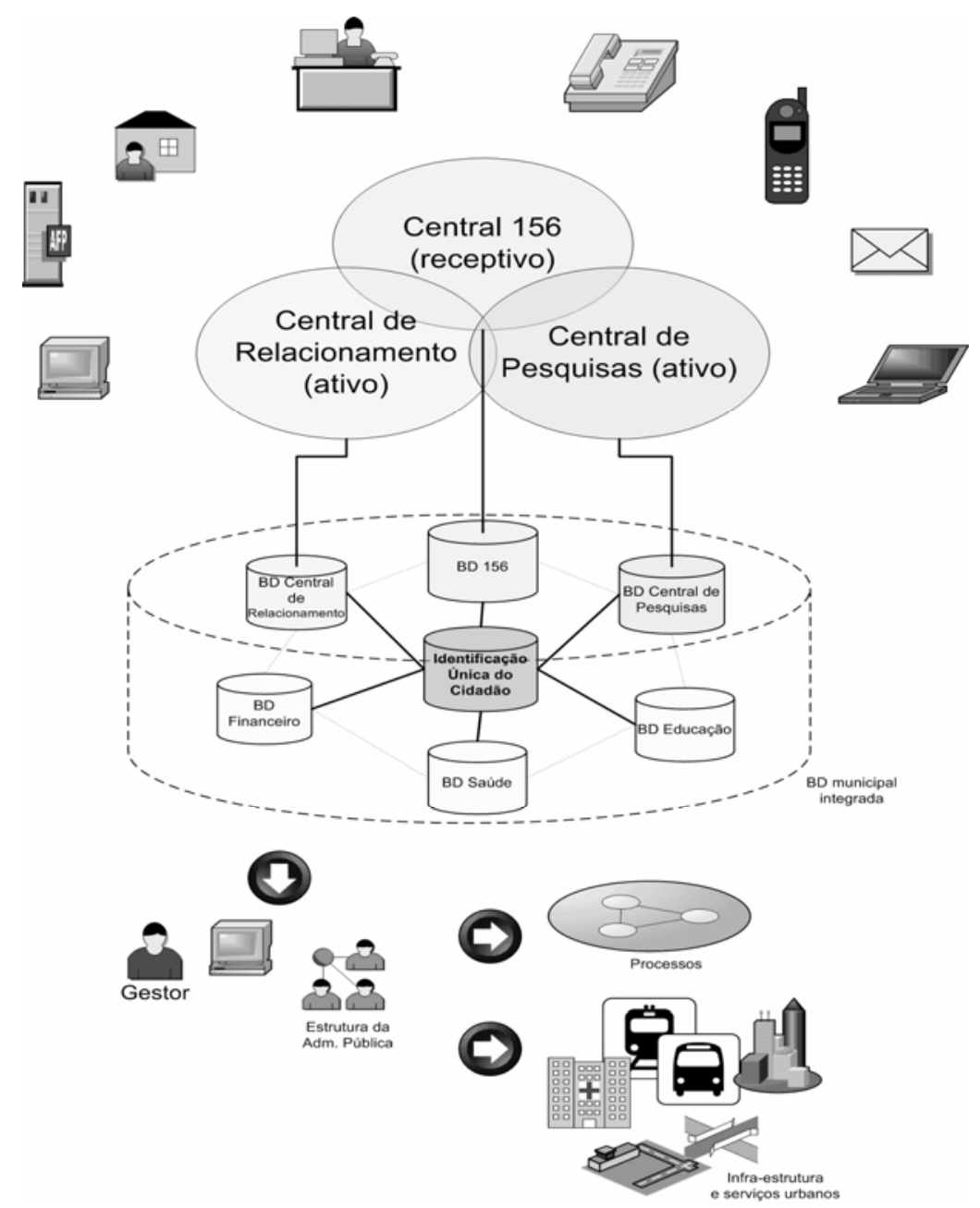

Figura 2 - Estruturação da proposta de CzRM Municipal

Existem algumas estratégias possíveis para a integração das diferentes bases de dados existentes no município. Poderia ser gerado um "grande” Data Warehouse, mas esta possibilidade não é muito atrativa quando são analisados os custos e o tempo de desenvolvimento de projetos deste tipo. Outra possibilidade, mais próxima à realidade financeira das Prefeituras e que pode trazer grandes benefícios ao sistema de informação municipal como um todo, é a geração de uma base central de dados de "Identificação Única do Cidadão”, que possui dados cadastrais básicos de todos os cidadãos, visando a identificação unívoca de cada cidadão e possibilitando o relacionamento destas informações cadastrais básicas do cidadão às suas informações nas demais bases de dados existentes na Prefeitura.

Deste modo, todos os Sistemas de Informação existentes na administração pública municipal devem identificar o cidadão relacionando suas bases de dados específicas a esta base de dados de identificação única do cidadão. Aliada à identificação do cidadão está a padronização dos dados em todos os Sistemas de Informação municipais, o que pode ser concretizado com a adoção de metadados, que são dados sobre dados. Estes metadados possuem a explicitação das informações que os sistemas tratam (como origem dos dados, formato dos dados, definições de negócios, ou atividades públicas, indicadores de qualidade dos dados, entre outros), e que são padronizados em toda a administração municipal e, em conjunto com a Identificação 
Única do Cidadão, viabilizam a implementação da comunicação e troca de informações entre diferentes sistemas. Permitindo assim, por exemplo, que em um atendimento da Central de Relacionamento que esteja alimentando a base de dados da Central de Relacionamento, seja possível identificar univocamente este cidadão e consultar rapidamente dados deste cidadão e seu histórico de relacionamentos financeiros com a Prefeitura na base de dados financeiros.

Ainda na Figura 2 estão representadas algumas das formas de interação com os cidadãos como telefone, carta, web, chats, e-mail, visitas a residências (agentes comunitários), quiosques de auto-atendimento e o atendimento em balcão.

O CzRM permite aos gestores públicos terem a sua disposição uma variedade de informações desde as demandas, as expectativas e os anseios até os níveis de satisfação e efetividade no atendimento das demandas solicitadas. Desta forma, o CzRM funciona como uma espécie de "motor" no processo de atendimento e de funcionamento da Prefeitura, sinalizando as ações para os diversos níveis hierárquicos; e, por outro lado, comunicando à população suas possibilidades de atendimento, seus projetos e obras de melhoria na infra-estrutura e nos serviços urbanos. Além disso, os gestores possuirão informações suficientes para avaliarem seus processos internos e os otimizarem buscando maior efetividade da máquina pública.

\section{Conclusão}

O sucesso de uma gestão pública municipal pode estar diretamente relacionado aos Sistemas de Informação e as tecnologias que serão utilizadas pelo gestor público, seu corpo técnico/gerencial e até na interação e disponibilização destas tecnologias aos cidadãos. O CzRM permite à Administração Pública conhecer seus cidadãos e prestar um atendimento personalizado com a gestão de todos os relacionamentos entre Prefeitura e cidadão.

Um grande desafio da implantação do Citizen Relationship Management (CzRM) Municipal é a mudança cultural necessária à organização. Mas o grande obstáculo tecnológico para a efetivação do CzRM Municipal é fazer com que as diferentes bases de dados existentes no município trabalhem de forma integrada e relacionada, problema este que pode ser tratado com a adoção de um sistema de identificação única do cidadão aliado à padronização de um metadados para a administração municipal.

A adaptação do CRM da iniciativa privada para a iniciativa pública, buscando contextualizar o cidadão como o cliente que tem expectativas e anseios pelos serviços públicos a ele prestados, pode representar uma aproximação da Administração Pública com o cidadão e um significativo avanço na otimização dos processos desta administração pública, apoiando inclusive o processo decisório na municipalidade.

A contribuição deste trabalho para a academia é o fomento de discussão da adaptação de conceitos e de modelos de gestão da iniciativa privada para o ambiente da administração pública, visando maior efetividade no atendimento aos anseios da população. Para as prefeituras serve como um modelo ou proposta inicial para a realização de um projeto de implementação de um CzRM, que pode vir a representar uma ferramenta fundamental para o planejamento e para a gestão dos serviços urbanos, 
uma vez que o CzRM possibilita conhecer o cidadão, trazendo a perspectiva, os desejos e a satisfação do cidadão para a análise do gestor público.

A principal limitação desse trabalho está relacionada ao fato de não ter sido encontrado em nenhuma Prefeitura a utilização efetiva de todos os elementos aqui expostos, inviabilizando um estudo de caso aprofundado. Uma pesquisa de campo mais aplicada junto a um município que utilizasse o CzRM com todos os elementos aqui expostos, possibilitaria uma análise dos impactos efetivos de uma solução como esta.

Como evolução deste trabalho poderia ser realizada uma busca mais extensa por municípios que estejam evoluindo para soluções similares ou que contenha alguns dos elementos aqui expostos, objetivando a realização de um estudo de caso aprofundado da utilização destes conceitos e das tecnologias envolvidas, para atender às necessidades da Administração Pública Municipal e de seus cidadãos.

\section{Referências}

BARZELAY, M. (2001). “The New Public Management: improving research and policy dialogue”, Regents of the University of California. California: Ucpress.

GIL, A. C. (2002). “Como elaborar projetos de pesquisa”. São Paulo: Atlas.

JONES, L. R.; THOMPSON, F. (2000). “Um modelo para a nova gerência pública”. Revista do Serviço Público, v. 51, n. 1, p. 41-79, jan/mar 2000.

LAKATOS, E. M.; MARCONI, M. A. (1991). “Metodologia Científica”, São Paulo: Atlas.

NACHMIAS, D.; NACHMIAS, C. (1987). "Research methods in the social sciences", New York:St. Martin’s Press

OSBORNE, D.; GAEBLER. T. (1992). "Reinventing Government: how the entrepreneurial spirit is transforming the public sector", Reading, MA: AddisonWesley.

REZENDE, D. A.; CASTOR, B. V. J. (2006). "Planejamento estratégico municipal: empreendedorismo participativo nas cidades, prefeituras e organizações públicas”, 2 ed. Rio de Janeiro: Brasport.

SCHELLONG, A. (2006). "Citizen Relationship Management: understanding, challenges and impact”. Proceedings of the 2006 international conference on Digital government research, pages 490-490. New York: ACM Press.

SILVA, E. L.; MENEZES, E. M. (2001). “Metodologia de pesquisa e elaboração de dissertação”, Laboratório de Ensino a Distância da UFSC, Florianópolis.

SWIFT, R. (2001). “CRM - Customer Relationship Management: o revolucionário marketing de relacionamento com os clientes”, Rio de Janeiro: Campus.

VERGARA, S. C. (2005). “Métodos de pesquisa em administração”, São Paulo: Atlas. 
ZENONE, L. C. (2001). “Customer Relationship Management (CRM) conceitos e estratégias: mudando a estratégia sem comprometer o negócio”, São Paulo: Atlas. 